\title{
Research on the Design of Medical Image Database System Based on Web
}

\author{
Liu Zhengliang ${ }^{1}$, LaiMin ${ }^{2}$ \\ ${ }^{1}$ Gannan Medical University, Ganzhou, Jiangxi, China, 341000 \\ ${ }^{2}$ Gannan Medical University, Ganzhou, Jiangxi, China, 341000 \\ hunter2011@foxmail.com
}

Keywords: Medical image database, Three-layer mode, Script language.

\begin{abstract}
In recent years, with the extensive application of database technique, medical image information becomes easier to be storage and archiving. Based on selection of development, script languagesand database technology, this paper designs the system structure, system process, webpage function, and system safety in order to provide an available and efficient design method of medical image database to supply some references for the related researchers.
\end{abstract}

\section{Introduction}

With the development of a large amount of medical image data, how to make use of these data to improve the efficiency has become an important subject. To solve the above problem, scientific researchers combine the advanced database technology, interactive technology and network technology and display a variety of medical image data on the screen. Users interact with each other, and through the network to share information in the world. Because of the accumulation of medical image data, it can greatly improve the medical clinical diagnosis level, and can provide a solid foundation for medical training, medical research and teaching, as well as the research and development of medical imaging. Therefore, the medical image databases in a large number of medical images are also followed by the gradual development of. Medical image database is essentially a multimedia database, but it is a web based database. Medical image database contains a variety of multimedia data types, and its image content belongs to medical category. The functions of medical image database include the organization, storage, query, display and data of the multimedia data, which are published in two aspects of Web. Storage and remote access of medical image data is important for a number of major projects, or between hospitals, research institutes and hospitals and research institutions. A perfect medical image data storage and remote access system will be more closely linked to each node, and can provide data sharing, information sharing, sharing of results and experience exchange. Therefore, it is necessary to establish a medical image database based on the web.

\section{Preparations of Design of Medical Image Database}

Selection of Development Environment. Network operating system is the core of the software platform. We use Microsoft Server Windows2000 as a unified server system platform. It has the following features: server hardware platform support, file and print sharing, suitable for distributed applications, it can be used in distributed application, which can be used to cross the other systems. The good security measure and advanced fault tolerance capability. Server as a database server supports multiple PC clients. The server has very good input and output performance and a lot of storage space, the application system of Client/server. IOOOM Ethernet switch is used to facilitate the information exchange. In order to guarantee the reliability, we consider the use of a PC machine as a data online backup server, to ensure that the main server operating normally. When the system is safe to switch to the backup,the server does not affect the normal operation of the system.

Selection of Script Languages. Script is the language that the application is expressed on the web. It can be embedded into the webpage. Usually the script runs on the server, but it can also be 
used for the client and server side. JavaScript is a scripting language based on object and event driven (Driven Event) and has security properties. The purpose of using it is to link multiple objects in a Web page with the HTML hypertext markup language, Java scripting language, and interact with the Web client. So as to develop the client application, etc. It is realized by embedding or transferred in the standard in HTML language. It appears to make up for the defects of HTML language, so JavaScript has been widely used in ASP. JavaScript has the following basic features. It is a script language JavaScript is a scripting language, it uses a small program to achieve the way of programming. Like other scripting languages, JavaScript is also an interpretive language that provides an easy development process. Its basic structure is very similar to $\mathrm{C}++, \mathrm{VB}$, Delphi and $\mathrm{C}$. But it is not the same as the language, we need to compile, but in the process of running program is progressive explanation. It combines with the HTML logo to facilitate the user's operation. JavaScript is a language based on object, and can be regarded as a kind of object-oriented. This means that it can use the object that you have created. Therefore, many functions can be derived from the interaction between the object's method and the script in the script environment. The simplicity of JavaScript is mainly reflected in the following: it is a simple and compact design based on the Java basic statement and control flow.

Selection of Database Technology. With the development of information technology, the way of computer processing data is changing from the early file management system to today's database management system. Microsoft SQL Server is a high performance server. Client/server Microsoft is capable of supporting the transaction processing, and also in the Windows NT Server Microsoft network environment management data access and development decision support applications. Also due to the SQL Server Microsoft is an open system, other systems can interact with it in good condition. Server SQL uses the C/S architecture to divide the work load into the tasks running on the server and the tasks running on the client. The client program is responsible for business logic and display data for the user, the client program is usually run on one or more clients, but it can also run on the Server SQL server. Server SQL manages the database and allocates the available server resources, such as memory, network bandwidth, and disk operation in multiple requests. It uses Transact-sql as a database query and programming language. Transact-sql is a structured Framed (Query Language), is a set of commands, allowing users to specify what information to obtain or change, using Transact-sql to access data, query, update and manage database system.

\section{Design of Medical Image Database System Based on Web}

Design of System Structure. As the Figure 1 shows, the structure of the system uses the browser/server/database three-layer modes. The first layer is the presentation layer. The system and the user to provide an interactive interface; the second layer is a functional layer, only for the administrator to carry out various management operations; the third layer is the data layer, the whole system is stored in all the data, this part is only used by administrators, ordinary users cannot be exposed to. The three layer structure of the server is divided into application server and database server two parts. The application server includes a portion of the application and a portion of the work from a dedicated server. This can make the client further smaller, the client only install the browser can access applications. The advantage of this three layer design scheme is that the client does not store data and program. It can improve the security and stability of the system, reduce the network traffic and reduce the maintenance quantity compared with the traditional $\mathrm{C} / \mathrm{S}$ structure. System user permissions assigned by users by the administrator through the management registered users, and users can use the presentation layer. When the system is running, the system management has the power to control the data in addition to the user's personal data. 


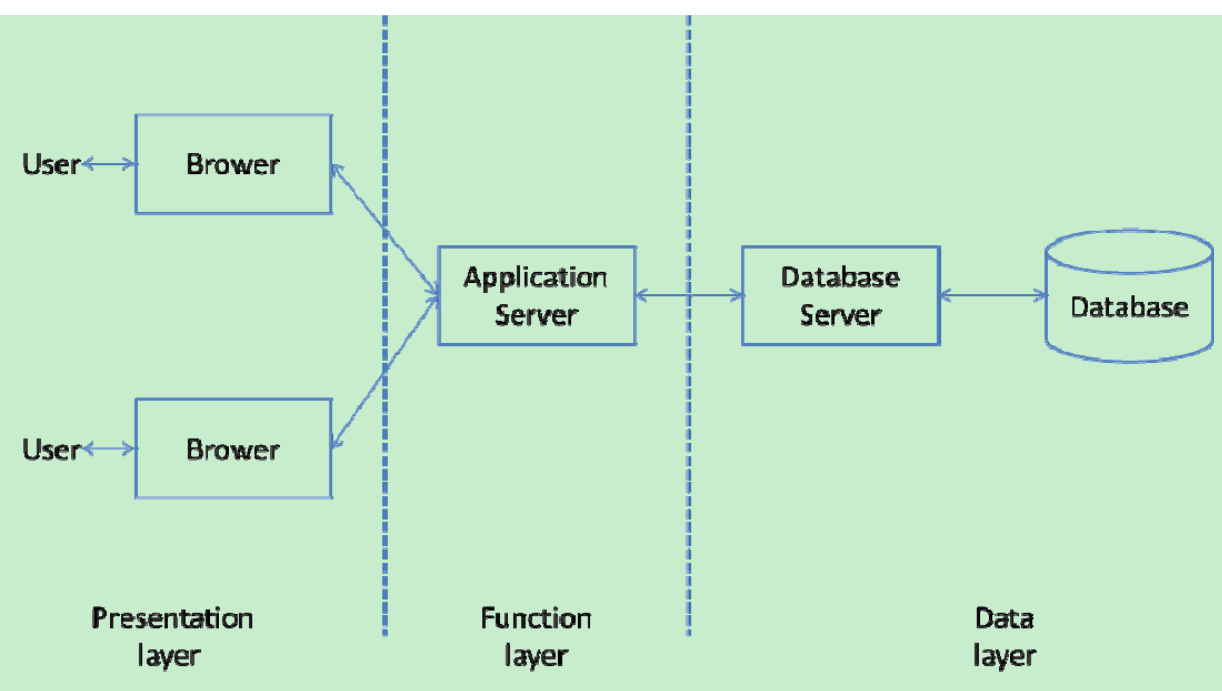

Fig. 1 Three-layer Frame of Medical Image System

Design of System Process. The whole system process can be divided into three parts. The first part is the module of users' login, which is composed of a user authentication database and a login system. The function of this module is for the user to log into the system, and according to different user levels to distinguish between different operating authorities. The second part is the human machine interface, the main component is a WWW server on the web interface, and function is to provide a platform for the user and the background of the database. The third part is the database, which contains a database for storing medical image data, which is run only as a background service. Users use the system as follows: the user first enters the login system to carry on the operation, in the process of logging in need of user database verification. If a validation error, the return of a user name does not exist or password error prompts, and returned to the user login window prompt re login. If verified through, then into the web page can be carried out a series of operations, such as browsing, query, update, etc., the premise is that the user has the appropriate operating authority. If the database is operated in the interaction field, the database returns the corresponding results to the user. Ordinary users can only use the database and cannot be changed, if the user is the database administrator, then the database will be saved and applied to the database. After the completion of the work, users can exit the system to finish the operation process.

Design of Webpage Function. A complete database system needs a beautiful webpages which are easy to operate. They are the front desk of the user's interactive operation interface. Administrators do not have to keep in front of the server, any place only need a computer to access the Internet to remote control system management. Administrator through special administrator account in the system of Web page to log in, access to the relevant management pages on the entire system can be operated, including Web page setup, user management, database update operation, etc.. In order to facilitate the management of remote administrators, the system provides a variety of upload services. The first is the use of no component upload mode, the administrator can be directly in the corresponding web page for data upload, and the use of the non-component upload method is the advantage of no need to install any plugin. Compared with the way in which the component is uploaded, the process of loading the plug-in is less, so it is faster, and it can also set up the file size, file type and so on, so as to ensure the stability of the database. This approach is suitable for a small amount of file upload. The second is the use of FTP.The administrator can transfer data to the database through FTP. This way is suitable for the uploading of large quantities of files, which is simple to realize using IIS (Internet information Server) function.

Design of System Safety. In terms of user management, we use a multi-level authority management strategy. Multi-level authority allocation principle is as follows: the first level is the system administrator level, second for senior user level, third for general users. System administrators have the highest authority. The database can be operated by different authority type allocation to second users (advanced users). The level can be obtained from the system 
administrator level permissions assigned to the general user. System administrators can also directly assign some authorities to the general operating personnel.

\section{Conclusion}

With the rapid development of various medical imaging equipment and computer technology, we can combine the two kinds of techniques to establish a medical image database system to improve the storage efficiency and maintenance management of medical image information. The relevant technology in the paper used to design the medical image database system based on web is comparatively mature. Web interface of the system is simple and the operations are easy to learn. It's able to meet the current needs through testing.

\section{References}

[1] Y.M. Li, Medicinal Animal Image Database Design and Implementation, Jilin University, 2015

[2] X. Shen, C.L. Wang, B.X. Hu, J.T. Wang, Exploration on the Establishment of Image Databases for Qinling Herbal Specimens in Chinese Herbal Medicine Museum, Journal of Shaanxi Col lege of Traditional Chinese Medicine, 5(2015)98-100.

[3] X.M. Wang, J.D. Xie, P.C. Wang, B. Han, Y. Zhang, W.S. Wang, J.F. Wang, Design and Implementation of Remote Diagnosis System of Medical Image Based On Web Technology, Chinese Journal of Physics, 5(2008)856-858.

[4] S. Jiang, The Design of Medical Image Database System Based on Web, Southern Medical University, 2007 